\title{
Fitting regression model and experimental validation for a high pressure PEM electrolyzer
}

\author{
M. Santarelli, P. Medina, M. Calì \\ Dipartimento di Energetica, Politecnico di Torino, Corso Duca degli Abruzzi 24, 10129 Torino, Italy \\ e-mail: $\underline{\text { massimo.santarelli@polito.it, pablo.medina@polito.it }}$
}

\begin{abstract}
Hydrogen production is the main obstacle today to develop a real future hydrogen economy. Research has focused many efforts in extracting it from clean and renewable sources. Different processes are analysed: photolysis, thermochemical cycles, algae, etc; these processes are still far from practical use. Electrolysis has represented the most studied and experimented area for obtaining hydrogen without employing fuel cracking. Nevertheless, for its practical storage, the hydrogen produced at low pressure needs to be mechanically compressed, with a high consumption of electric power. Advanced materials and improved design allow to obtain hydrogen from electrolysis directly at medium-high pressure (70 bar) with no need of mechanical compression stages. This single-step process is more efficient than the twostep electrolysis + mechanical compression process. In this paper the authors display the experimental results obtained with a prototype of high pressure PEM electrolyzer manufactured by Giner Electrochemical Systems LLC, including the description of the test bench for the experimental characterization. The experimental design, based on Design of Experiments techniques, studied the effect of the main operation factors (temperature, pressure, water flow) at different levels of power load, presenting a regression model of the electrolyzer voltage as a function of the operating factors, at different values of the electric load.
\end{abstract}

\section{Keywords}

hydrogen production, PEM electrolyzer, high pressure electrolysis, design of experiments

\section{Introduction}

Commonly used electrolyzers (usually alkaline, which require frequent maintenance that includes disposal and replacement of the highly caustic electrolyte) generate hydrogen at relatively low pressures. Furthermore, hydrogen has still to be compressed for storage. One solution to this problem is to use a compressor to increase the hydrogen pressure. However, the energy investment required to compress hydrogen, as well as the maintenance of hydrogen compressors, makes this option very expensive for large-scale application of this technology. The dependence on a mechanical compression stage penalizes the whole efficiency, and becomes more relevant when elevated pressures are required.

The alternative is to compress hydrogen electrochemically, pulling protons across the electric field to a pressure-controlled cathode camera. It has been observed that the energy that protons need to move across a pressure gradient is much smaller than the power supplied to a multi-stage compressor. Some studies [1] reveal that, at elevated pressures, a considerable fraction of water vapour present in the produced hydrogen gas undergoes condensation. This reduces the cost of desiccating the gas, because at elevated pressures the gases are virtually free of moisture. There is one more, implicit, advantage of electrolysis at elevated pressures, namely, the electrolysis can be run at temperatures above $100^{\circ} \mathrm{C}$, which substantially reduces the cell voltage. Of 
course, electrolysis at elevated pressure has some drawbacks, too: the effect of pressure on the process thermodynamics, and on the cell overvoltages, causes an increase in the theoretical and actual voltage of water decomposition. Moreover, the electrolyzer design becomes more complicated, and the requirements imposed on the electrolyzer strength and air tightness become more severe.

The trend today for future electrolysis devices, even at high pressure, goes through PEM technology. many studies and papers [2,3] demonstrate the interest in this technology. The PEM electrolyzer uses a solid electrolyte membrane that can be expected to increase the lifetime of the electrolyzer. No caustic alkaline or acidic fluid electrolyte is required. Additional advantages of PEM electrolysis over alkaline electrolysis include lower parasitic energy losses and higher purity hydrogen output. PEM electrolysis is potentially a simple, sustainable, and cost-effective technology for generating, compressing, and storing hydrogen.

Few Authors refer to experimental research for PEM electrolysis. Schug [4] describes the operational characteristics of high pressure electrolysis, analyzing the responses of the main systems parameters and how the material problems as corrosion can be solved. Grigoriev et al. [3] make a general overview of PEM electrolysis and its possible applications including the obtained polarization curves for different catalyst compositions and different operation temperatures and pressures; also, they have developed [1] a mathematical model and relevant software, which allow to model the polarization curves of a PEM-based electrolyzing cell on the basis of literature and experimental data. Görgün et al. [5] describe a dynamic model for PEM electrolyzer based on conservation of mole balance at the anode and the cathode, including water transport, electro-osmotic drag and diffusion through the membrane; also hydrogen storage dynamics are presented. Choi et al. [6] also have developed a simple model based on Butler-Volmer kinetics for electrodes polarization, and considering also the transport resistance in the polymer electrolyte. Onda et al. [7] have made a detailed comparison between atmospheric and high pressure electrolysis efficiency, with an analytical study of the effect of enthalpy and Gibb's free energy variation with stack temperature or pressure. Barbir et al. [8] introduce the possibilities of integrating a electrolyzer with a PV array in a isolated or grid connected layout, displaying the results of efficiency for different pressures and performance duration against time. Marshall et al. [9] have developed new electro catalytic materials used for the oxygen evolving electrode, making great improvements in efficiency, presenting their results with steady state polarization measurements and electrochemical impedance spectroscopy. Roy et al. [10] have performed a comparison of total energy consumption for electrolysis at various operating pressures up to $700 \mathrm{~atm}$, including practical considerations such as corrosion, hydrogen embrittlement, operational complexity, dynamic response and cost.

In this paper, the Authors display the experimental results obtained with a prototype of high pressure PEM electrolyzer manufactured by Giner Electrochemical
Systems LLC (US), including the description of the test bench for the experimental characterization. The objective of this paper is to analyse the influence of different operation factors on the voltage (and consequently the electric power) supplied to a high pressure PEM electrolyzer at different levels of stack current (or hydrogen production).

Several polarization curves have been obtained in different conditions, displaying the trend of the stack voltage against current loaded from the electrolyser. The experimental design, based on Design of Experiments techniques, studied the effect of the main operation factors (temperature, pressure, water flow) at different levels of power load. The experimental data has been processed using a factorial design (Yates' technique); using the analysis of variance (ANOVA): the factors have been selected in two extreme operational levels compounding a $2^{3}$ experimental plot. The results of the tests have been displayed comparing the effect of the factors in terms of single or combined effects.

Finally, a regression model of the electrolyzer voltage is presented, as a function of the selected factors, providing an analytical expression of the behaviour of the device at different operating conditions. The final aim is to optimize the efficiency of the hydrogen production at different pressures.

\section{Description of the equipment}

\subsection{Stack}

The stack utilised for the tests is the prototype Giner GS10 electrolyzer provided by Giner Electrochemical Systems LLC (US). The stack produces hydrogen at a mid-high pressure (max 70 bar), and oxygen at low pressure ( $\max 3$ bar). Maximum hydrogen output production is $0.1 \mathrm{~kg} / \mathrm{h}\left(1.1 \mathrm{Nm}^{3} / \mathrm{h}\right)$, while oxygen is produced in a half volume rate $\left(0.55 \mathrm{Nm}^{3} / \mathrm{h}\right)$. Power loaded by the stack at maximum production rate is 5.6 $\mathrm{kW}$. The electrolyzer is made of 12 planar cells, with a exchange surface of $160 \mathrm{~cm}^{2}$. The main characteristics of the electrolyzer are detailed in Table 1.

\begin{tabular}{|l|c|}
\hline \multicolumn{1}{|c|}{ Data } & Value \\
\hline Temperature & $50 \div 60^{\circ} \mathrm{C}$ \\
\hline Anode pressure & $0 \div 3.5 \mathrm{bar}$ \\
\hline Cathode pressure & $0 \div 35 \mathrm{bar}$ \\
\hline Water flow & $>5.5 \mathrm{l} / \mathrm{min}$ \\
\hline Water resistivity at $25^{\circ} \mathrm{C}$ & $>1 \mathrm{M \Omega} \mathrm{cm}$ \\
\hline Maximum $\mathrm{H}_{2}$ in $\mathrm{O}_{2}$ content & $0.5 \% \mathrm{vol}$ \\
\hline Maximum $\mathrm{O}_{2}$ in $\mathrm{H}_{2}$ content & $0.5 \% \mathrm{vol}$ \\
\hline Maximum $\mathrm{H}_{2}$ production rate & $1.1 \mathrm{Nm}^{3} / \mathrm{h}$ \\
\hline Current at max. prod. rate & $224 \mathrm{~A}\left(\mathrm{at} 1.4 \mathrm{~A} / \mathrm{cm}^{2}\right)$ \\
\hline Voltage at max. prod. rate & $25 \mathrm{~V}$ \\
\hline Power at max. prod. rate & $5.6 \mathrm{~kW}$ \\
\hline Voltage at recommended current & $22 \mathrm{~V}(\mathrm{at} 6.89 \mathrm{bar})$ \\
\hline $\mathrm{H}_{2}$ prod. rate at recom. current & $0.4 \mathrm{Nm}^{3} / \mathrm{h}$ \\
\hline
\end{tabular}

Table 1. Electrolyzer operational characteristics

It has a compact size, as it can be seen in Figure 1, with an external diameter of about $25 \mathrm{~cm}$. It has four fluid connections (water in, water+oxygen out, hydrogen out and nitrogen in) and two electrical poles (anode-cathode) 
with two wire connections each for optimal current conduction.

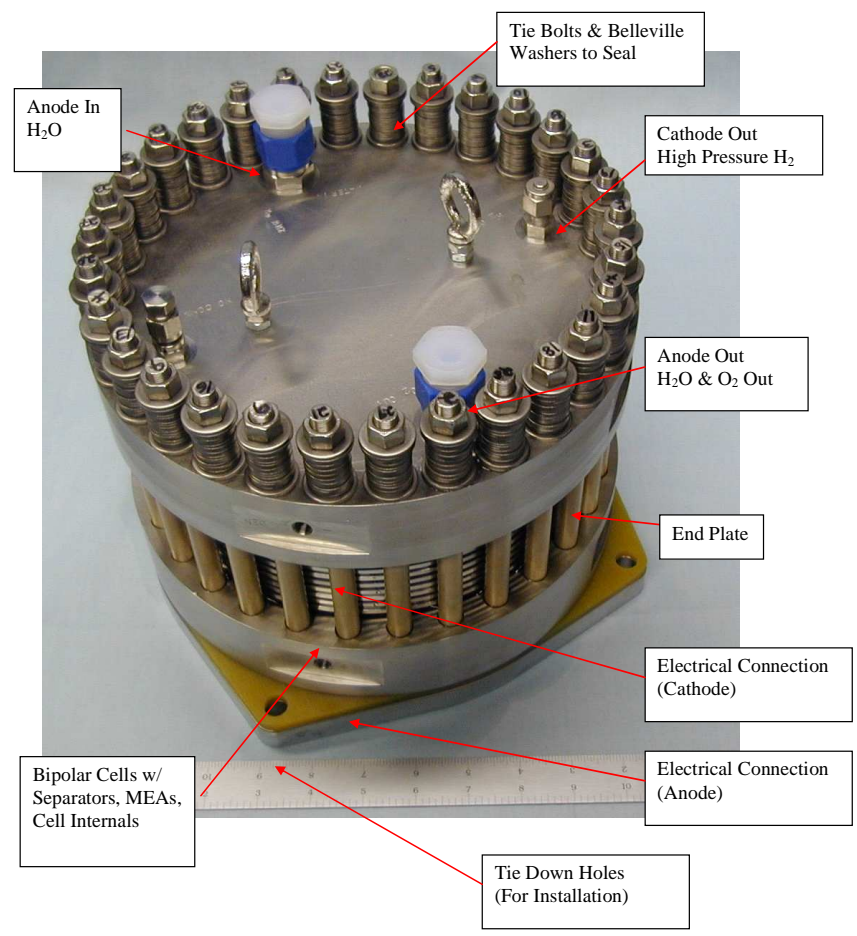

Figure 1. High pressure PEM electrolyzer tested

The cell stack, installed with vertical axel, is contained between two terminal heads (end plates, or end covers); the cathodic (superior head) and the anodic (inferior head). Heads are linked with ties, which functionality is to compress the fittings between the cells, keeping sealed the internal fluids. Each tie is supplied with some tongs, capable of compensating the dilatations of the stack, keeping the accurate pressure in the cell sealing. The ties are electrically isolated from the anodic head, which is also isolated from the base plate where the stack lays. The connectors are set in the cathodic head, where the inflow and outflow channels arrive.

During operation, the anode side is kept at nearly ambient pressure, while the cathode side is maintained at pressure up to 70 bar by a back pressure valve. The hydrogen is pushed electrochemically across the membrane to a much higher pressure: the cathode pressure rises just from the gas outflow produced by electrolysis. Thus, the electrolyzer works with a elevated pressure gradient between electrodes, which are therefore designed to support the mechanical stress and to assure the gas tightness to block any dangerous leakage from cathode to anode.

In the Figures 2 to 4 the main characteristics of the electrolyzer in stationary operation conditions are represented.

First, In Figure 2 the stack cells polarization curves for different operating conditions are shown: the main factors varying system output are water temperature (considered at the entrance of the stack) and cathode pressure; the stack has been tested in the extreme values allowed for these factors. As expected, the polarization increases when low temperature or high pressure conditions are set, which means worse results in terms of efficiency.

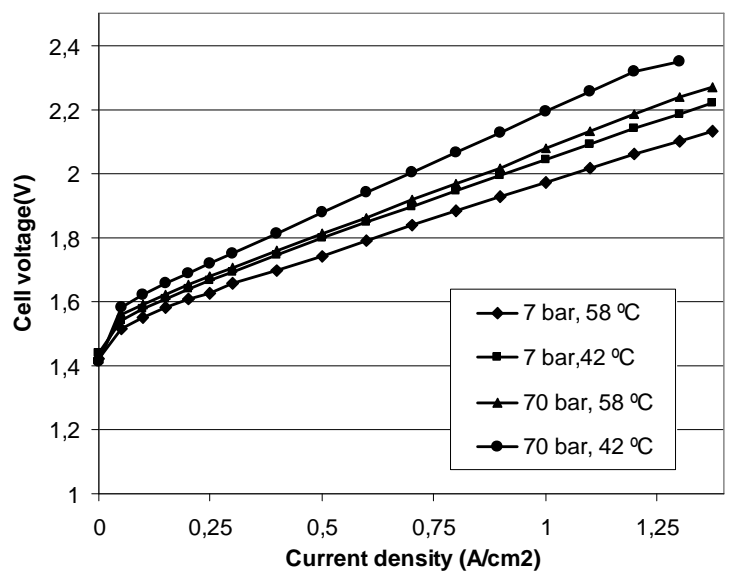

Figure 2. Cell polarization curves in different operating conditions

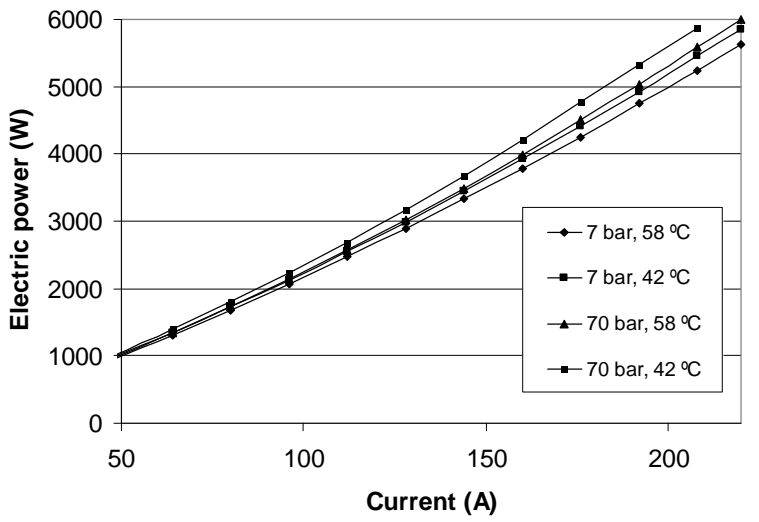

Figure 3. Electric power loaded from the stack in different operating conditions

In Figure 3 the stack power is shown. Lower stack currents not have been represented because power loaded is almost the same in any condition. As we can observe, maximum power input is limited at around $6 \mathrm{~kW}$. At maximum power loaded, the difference between the most favourable condition $\left(7 \mathrm{bar}, 58^{\circ} \mathrm{C}\right)$ and the worst one $(70$ bar, $42^{\circ} \mathrm{C}$ ) is around $10 \%$, that is around $500 \mathrm{~W}$ difference.

In Figure 4 the specific work consumed by the stack for different production rates and different conditions of pressure and temperature are shown. The specific work rises when operating with lower temperatures or higher pressures: the trend is the same of the voltage, because hydrogen production rate is directly linked with stack current. At high production rates, the specific work is noticeable when increasing pressure, even if this effect is significantly mitigated by the increase of temperature: the values are around $60 \mathrm{kWh} / \mathrm{kgH}_{2}$ at 70 bar. At low pressure the values are known, around $55 \mathrm{kWh} / \mathrm{kg} \mathrm{H}_{2}$. 


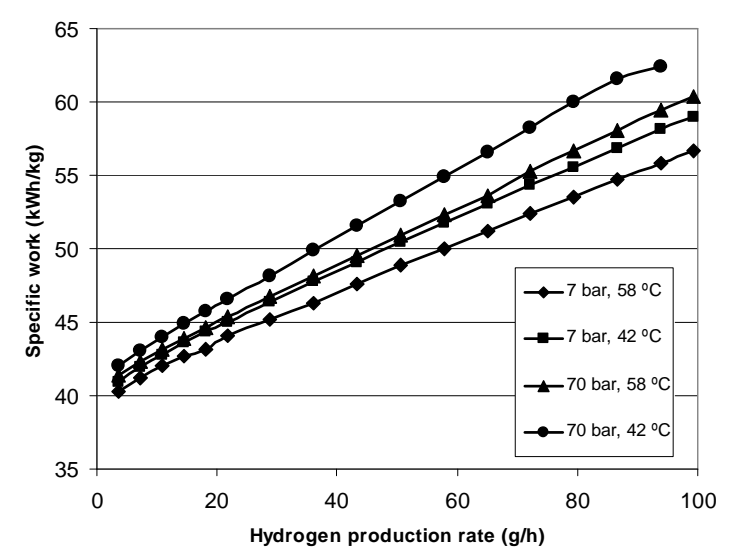

Figure 4. Specific work against hydrogen production rate in various operating conditions

\subsection{Test bench}

The test bench has been designed by Politecnico di Torino in collaboration with Sapio S.p.A. (Italy). A schematic flow of the test bench is shown in Figure 5. Its function is to supply the electrical power and the process fluids in order to ensure the electrolyser stack operation. It allows to control several physical parameters, and the measurement of many output data. Particular emphasis has been devoted in the design in order to ensure the safe operation of the electrolyzer. In fact, the polymeric membrane has a permeability to hydrogen and oxygen; due to the high pressure gradient from cathode to anode, this driving force could push hydrogen from cathode to anode across the membrane and a dangerous mix with oxygen could occur; this concentration must always be kept below a safety level. The test bench must therefore detect the hydrogen-in-oxygen concentration and stop operation in case of potential danger: the operation stop has been imposed equal to $0.5 \%$ of hydrogen-in-oxygen concentration. Moreover, all the working conditions have to be measured in order to avoid damages to the stack, for example due to overload.

The test bench is made up of four main subsystems. First, the water supply system, which sends a demineralised water flow into the stack for electrochemical reaction and for cooling, while taking away the oxygen bubbles produced in the anode. Second, there is the hydrogen circuit, which delivers the high pressure gas to the separation vessels where water is removed from hydrogen. Third, the nitrogen supply system is applied to inert any flammable mix inside the ducts and to purge the system before activation.

Finally, there is the electrical power supply, regulated from a AC/DC voltage regulator driven from the control panel. The regulation adjusts the source voltage to the characteristic curve of the electrolyzer for any current selected.

\subsection{Control panel}

The control system is based on a PLC (Programmable Logic Controller) which acquires all the information provided by sensors and transducers, and controls the actuators in order to impose the desired values to the controlled parameters. The PLC is also responsible for system safety, having to shut down the system in case of detection of anomalous operation. The control system is interfaced with a computer, which main control window is displayed in Figure 7. All measured parameters can be checked easily by operator. Finally, the data acquisition system, records all the running parameters of the process in a file for its analysis. The acquired data can be shared with remote workstations.

The measured quantities are: electrical data (current, voltage), temperature data (water in and out the stack, hydrogen), pressure data (hydrogen, oxygen and nitrogen, differential pressure between anode inlet and outlet), levels in water separators and other data as water conductivity and water flow. All the control devices are installed in the electrical module, which also carries the control devices.

\section{Methodology of the experiments}

The experiments have been programmed following a factorial design based on the Design of Experiment technique (Yates method). This technique allows to perform a parametric analysis on the electrolyzer operation by evaluating if a factor (independent variable) has a significant effect on the output parameters (dependent variables) or its effect is negligible. A positive main effect of a factor means that, when the factor is fixed at its upper level, it causes an increase of the dependent variable.

In this work the experiment has been designed using a $2^{3}$ factorial problem. The three factors chosen have been: temperature of the stack (considered water inlet temperature), cathode pressure (or hydrogen outlet pressure) and demineralized water flow. The eight treatments that will be analyzed $\left(2^{3}\right.$ factorial $)$ can be displayed geometrically as a three dimension cube.

The designed treatments have been performed with completely randomized design and with two repetitions for each treatment in order to analyze the simulated data with an analysis of variance (ANOVA). The treatments have been performed for different current levels of the stack obtaining a complete polarization curve for each combination of the other three variables. 


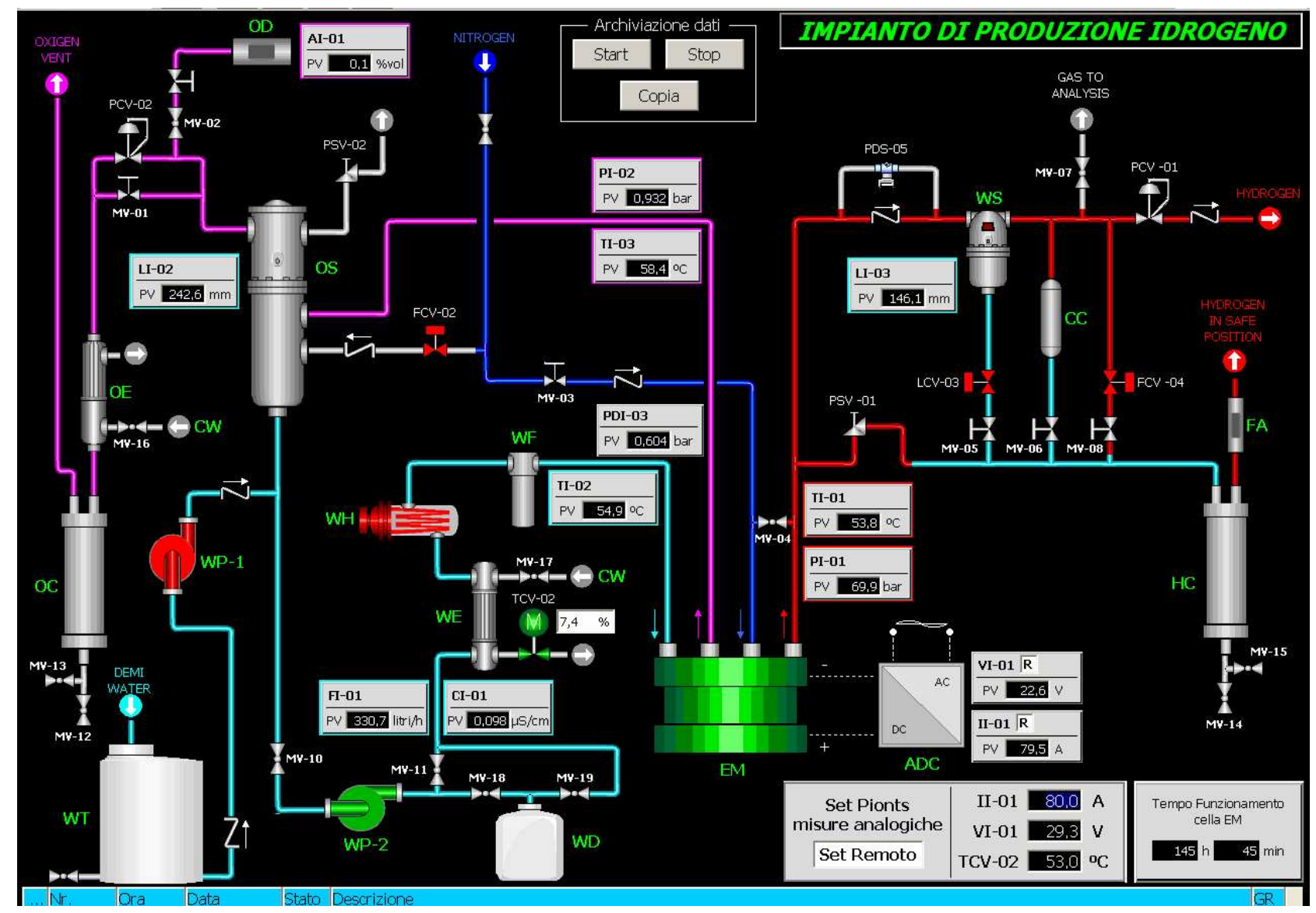

Figure 5. Display of the main control window of the test bench

With this design of experiments it is possible to obtain first-order regression models for the investigated dependent variables in terms of the significant factors. If higher-order regression models would be obtained, then the experimental campaign should be designed by using a different approach (i.e. $2^{k}$ factorial analysis with Central Composite Design CCD for the second-order regression models or $3^{k}$ factorial analysis). The first-order regression has been assumed as sufficient for a first screening of the operation of the system.

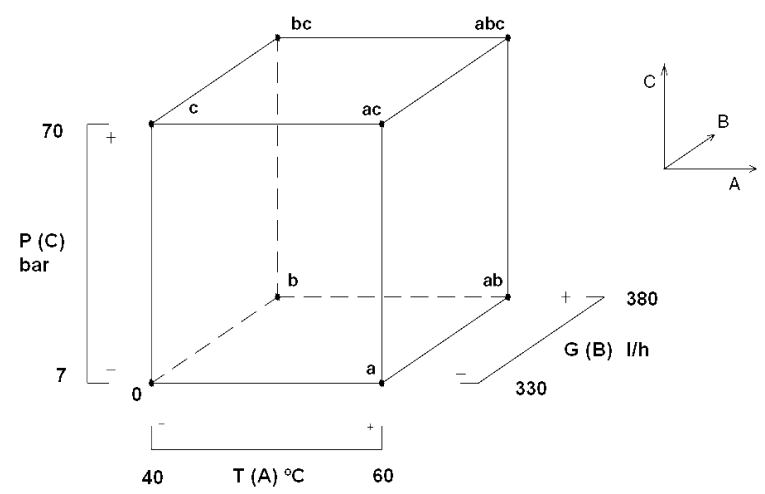

Figure 6. Yates' treatment experimental plot
The aim of this sequential approach is to obtain analytical relations between the dependent variable (the average stack voltage) and the analyzed independent variables (hydrogen pressure, stack temperature, water flow and current). The regression models allow to represent multiple responses of the stack operation by plotting contour plots and response surfaces. Moreover, they allow to apply constrained optimization methods in order to maximize one or more dependent variables (i.e. DC supplied power, hydrogen production) at different current levels. Once the input factors have been chosen, it is fundamental to determine their range of variation (the experimental domain). The range of the factors is shown in Figure 6.

In the tables and figures presented in the paper, the regression models are written in coded forms, where the independent variables varies between the values -1 (corresponding to the lower bound of its experimental domain) and +1 (corresponding to the upper bound of its experimental domain). Nevertheless, in the analytical regression models the coefficient linking the independent and dependent variables are not a-dimensional, because they represent the sensitivity coefficient linking these variables when the regression models are expressed in the physical form. Therefore, the unit measures are consistent in a regression model due to the procedure applied to obtain them. The models can be expressed in physical form with a simple manipulation of the equation. 


\section{Results and discussion}

Analysis will be displayed for two levels of current: the lowest value is related to a current density of $0.1 \mathrm{~A} / \mathrm{cm}^{2}$ (corresponding to $16 \mathrm{~A}$ to the stack) and the highest value is related to a current density of $1 \mathrm{~A} / \mathrm{cm}^{2}(160 \mathrm{~A})$. Two extreme current levels have been selected to characterize the electrolyzer operation in completely different load conditions, as transport and thermo-chemical phenomena vary significantly inside the cell membranes from case to case.

The analysis is divided in two parts: the first part focus on the analysis of the experimental data recorded, in particular the significance of the effect of every factor on the dependent variable (voltage), and the residual analysis. The second part consists in the identification and selection of the best regression model, obtained from the experimental data and used as a prevision model. The factor are codified to simplify the result tables and plots. Factor $a$ represent the stack temperature, factor $b$ the cathode pressure, factor $c$ the water flow; each combination of factors is expressed as a product of their letters.

First, we evaluate the significance of the effect of the three operation factors and their combinations on the stack voltage, comparing the results for low and high stack current density. The effect plot reveals us how every factor modifies the output variable. The red line represents the minimum threshold of significance, also given by the algorithm criteria.

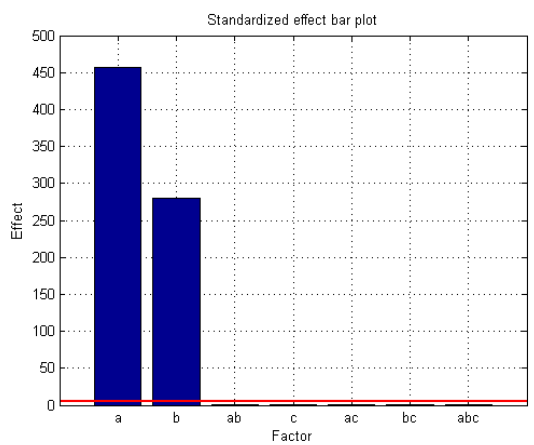

\section{$i=0.1 \mathrm{~A} / \mathrm{cm}^{2}$}

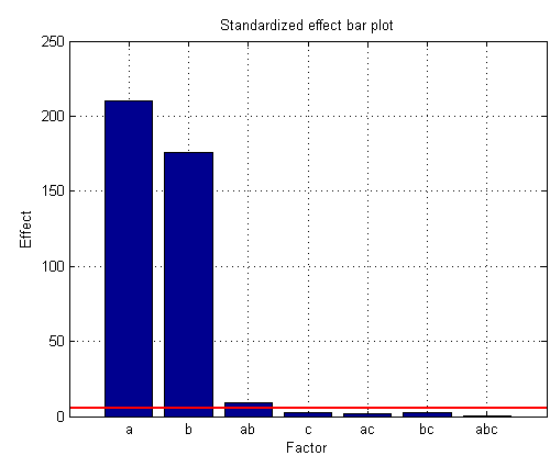

$i=1.0 \mathrm{~A} / \mathrm{cm}^{2}$

Figure 7. Effect of each factor on the output variable

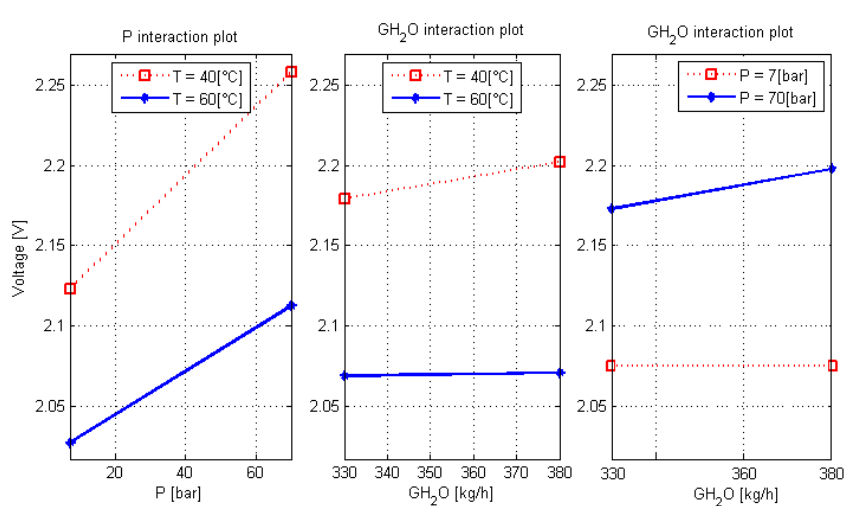

a) $0.1 \mathrm{~A} / \mathrm{cm}^{2}$
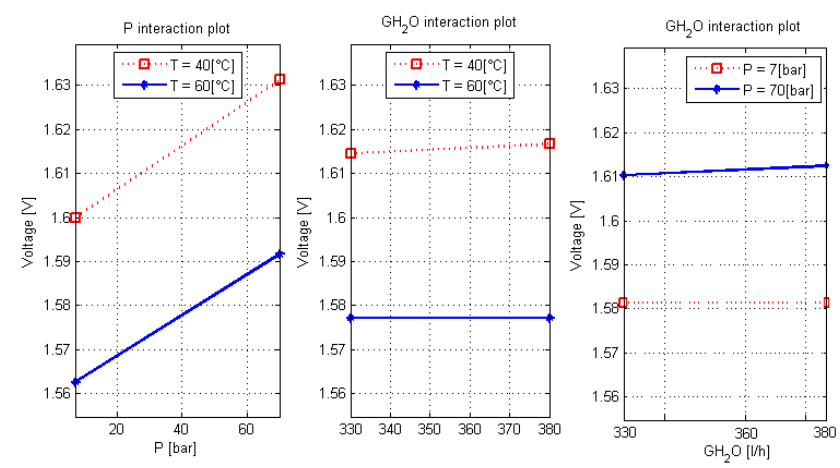

b) $1 \mathrm{~A} / \mathrm{cm}^{2}$

Figure 8. Interaction effect of two factors in the cell voltage

In Figure 7 we can see that at low current, temperature has a preponderant effect, almost doubling the pressure. Other factors seem to be negligible. At high power, the pressure effect rises almost up to the temperature level. Unlike the first case, now it is noticeable the effect of the interaction of these two factors.

The Figure 8 shows the increment of the cell voltage varying one factor, while the second is kept at its extreme values and the last remains fixed. They are called interaction plots, because they detail the output parameter value for two factor combination. At low current rate, the lines are almost parallel, that means that the effect produced from the abscise factor is similar in both extreme values of the other factor displayed in the legend. This means that the interaction is weak. We can also notice the negligible effect of water flow through the stack, especially at high temperature and low pressure.

In Figure $8 \mathrm{~b}$ we can see the interaction plot at high power loaded from the electrolyzer. In this case the lines are clearly not parallel: in particular, the effect produced by the pressure is very influenced by the value of temperature, producing a high interaction between them. As the previous case, the effect of water flow at low pressure or high temperature is completely zero. Temperature has a large effect on the voltage increase caused by pressure variation: when varying cathode pressure between its limits (7-70 bar), at low temperature the cell voltage increases up to $35 \%$ more than at high temperature. This value can be observed in the left graph of Figure 8b, looking at the interval between lowest and 
highest point in both lines, that is also the different curve slope for both cases.

After the data analysis, we can proceed to build a model based on these results, obtaining a regression function fitting the experimental points. A self-made program selects the best model and displays the obtained analytical expression for both current densities:

\begin{tabular}{|c|}
\hline Analytical regression model \\
\hline For low current density $\left(0.1 \mathrm{~A} / \mathrm{cm}^{2}\right)$ \\
\hline$V=1.67-1.9 E-3 T+4.8 E-4 P$ \\
\hline For high current density $\left(1 \mathrm{~A} / \mathrm{cm}^{2}\right)$ \\
\hline $\begin{array}{c}V=1.94+2.88 E^{-3} T+9.2 E^{-4} p-3.968 E^{-5} T \cdot P+ \\
+9.86 E^{-4} G_{H 2 O}-2.1 E^{-5} T \cdot G_{H 2 O}+7.94 E^{-6} P \cdot G_{H 2 O}\end{array}$ \\
\hline
\end{tabular}

Table 2. Regression models for low and high stack current analysis

In addition to the factor coefficients, the algorithm returns the confidence interval at $95 \%$ of the obtained value. This is obtained considering the experimental error measured between both replications of every test.

We can observe first that much more regressors have been chosen for the high current model. Obviously, they are different because, as shown in Figure 8, at high current density the interaction of temperature and pressure becomes significant.

Once evaluated the regressor model which best fits the experimental results, it is possible to represent the contour plots, which simulate the electrolyzer operational performance. 3D graphics (surface plots) are also available, but the contour plots are more clear.

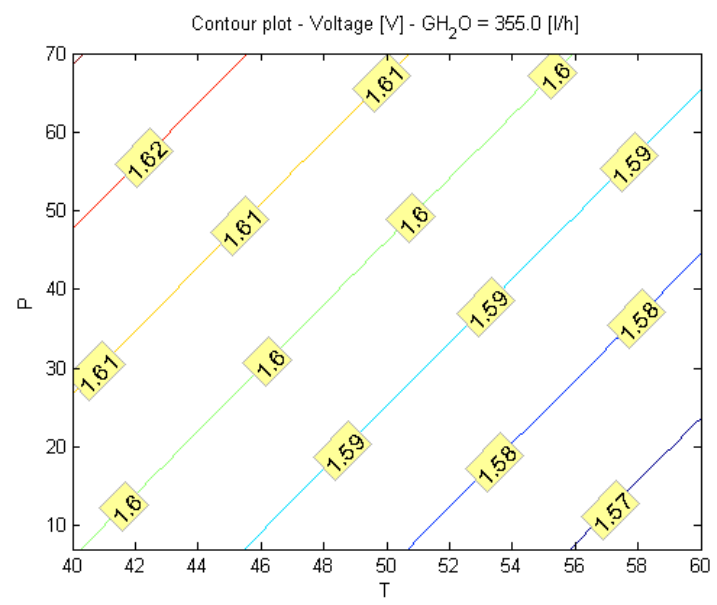

In Figure 9a the contour plots obtained for low stack current density are shown. Pressure and temperature are the only significant factors (the water flow is negligible), as noticed with the first data analysis. In the p-T contour plot it is shown that the variation of cell voltage is similar when rising pressure or reducing temperature (the level lines displayed are almost diagonal to the axis).

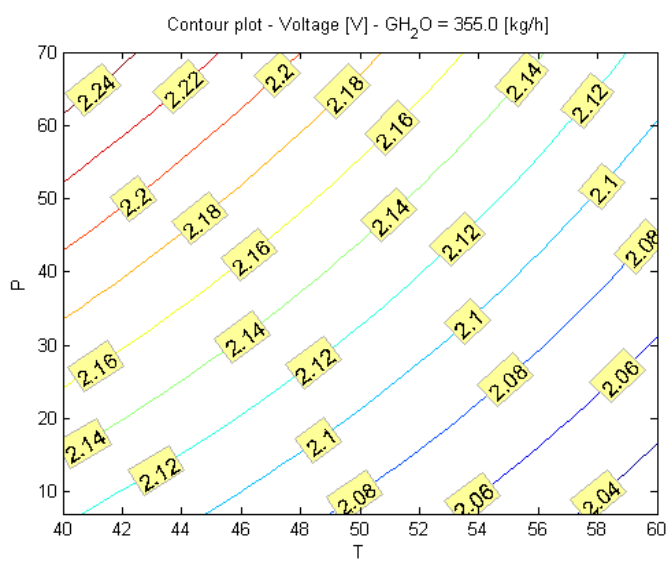

a) $0.1 \mathrm{~A} / \mathrm{cm}^{2}$
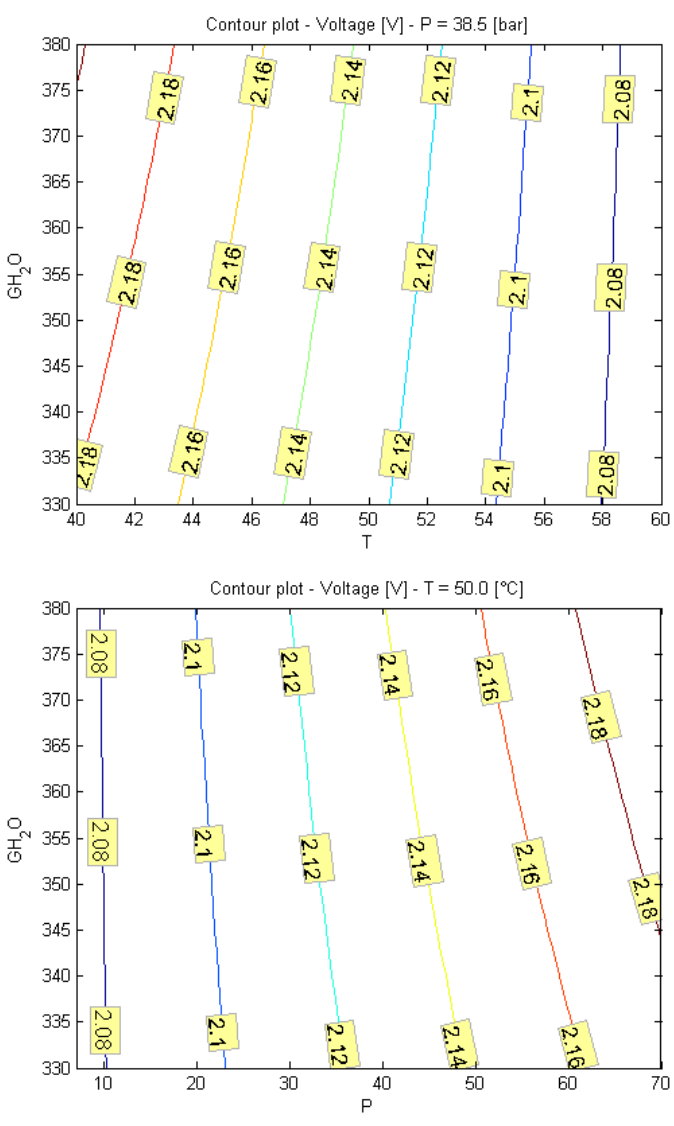

b) $1 \mathrm{~A} / \mathrm{cm}^{2}$

Figure 9. Cell voltage dual factor contour plots

In case of high current density (Figure 9b), it is noticeable that there exist a curvature on the voltage plot. This is linked to the fact of having the $\mathrm{p}^{*} \mathrm{~T}$ interaction term in the regression model. Therefore, especially at high current density, it is very important to have the possibility to operatre at high values of temperature, to reduce the effect of the pressure in the voltage increase. Other plots reveal that, at high current densities, the water flow rate seems to have an effect at lower temperatures and higher pressures. This trend is generated from the negative term $\mathrm{T}^{*} \mathrm{G}_{\mathrm{H} 2 \mathrm{O}}$ and the positive term $\mathrm{p}^{*} \mathrm{G}_{\mathrm{H} 2 \mathrm{O}}$ in the regression curve. Nevertheless, the effect of the water flow rate has been demonstrated to be not significant in the analysis of the effects, this effect may be associated with the experimental randomized error, then it can be neglected in our statements. 
After having a look at all the graphs, we can conclude that temperature and pressure have a predominant effect in both cases, remaining the voltage almost invariable with the water flow in the stack. We can resume this in Figure 10, where the main effects for temperature, pressure and water flow are displayed. Results have been plotted for $1 \mathrm{~A} / \mathrm{cm}^{2}$ for clarity. This plots are obtained keeping invariable the other factors so they give us an idea of the significance. To get an idea of the importance of each factor, we can say that at higher current densities $\left(1 \mathrm{~A} / \mathrm{cm}^{2}\right)$ :

- temperature causes a variation in cell voltage of $5.25 \cdot 10^{-3} \mathrm{~V} /{ }^{\circ} \mathrm{C}$ at 7 bar and a variation of $7 \cdot 10^{-3}$ $\mathrm{V} /{ }^{\circ} \mathrm{C}$ at 70 bar approximately;

- $\quad$ pressure produces a variation of $1,6 \mathrm{E}-3 \mathrm{~V} / \mathrm{bar}$ at 60 ${ }^{\circ} \mathrm{C}$ and $2.1 \mathrm{E}-3 \mathrm{~V} / \mathrm{bar}$ at $40{ }^{\circ} \mathrm{C}$ approximately;

- water flow sent to the stack produces a variation of $4 \mathrm{e}-4 \mathrm{~V} / \mathrm{l} / \mathrm{h}$ in cell voltage at higher pressure and lower temperature.
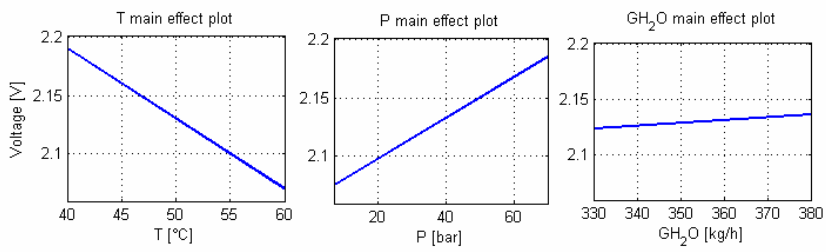

Figure 10. Plot of the main effects of the three factors for 1

$$
\mathrm{A} / \mathrm{cm}^{2}
$$

These values confirm the negligible effect of water flow in the stack working output compared to the other two factors, which have an almost equivalent importance in the sensitivity of the cell voltage.

\section{Conclusions}

The aim of this paper was to display the results of an experimental design analysis of a high pressure PEM electrolyzer. The results of the Yates' Technique experimental design have been presented.

Two analysis have been held at extreme stack operational conditions: 0.1 and $1 \mathrm{~A} / \mathrm{cm}^{2}$. The goal of the laboratory tests has been to make an analysis of the significance of the tested factors (stack temperature, cathode side pressure, water mass flow at the anode) on the dependent variable (stack and single cell voltage) and a quantification of their effects. A further results has been to obtain the analytical models that predict the electrolyzer operation in several conditions, in order to report the electrolyzer contour plots.

Temperature and pressure have a predominant effect in both cases, remaining the voltage almost invariable with water flow in the stack. For low and high power loaded from the stack, the temperature of the water inflow represent the most significant variable on the cell voltage, reducing the power load when the stack is operating at higher temperatures.

Also, it has been displayed the non constant effect of pressure at different operational temperature, producing bigger jumps in cell voltage when working at low temperature. It would be advisable then to run the electrolyzer at higher temperatures to alleviate the negative pressure effect in efficiency. Therefore, the pressure increasing trend of new electrolyzer models would need higher operating temperatures to reduce the fall of performance. Further studies should be done to improve the membrane thermal robustness to ensure operation over $60^{\circ} \mathrm{C}$, which will allow higher efficiency of the hydrogen production system.

In particular, that results for high current analysis should be especially considered because of the practical interest. In fact, these machines are designed to operate at high current densities for better products purity and system safety, and to optimize the investment.

\section{Acknowledgements}

This work was carried out as a part of a large project financially supported by the Ministry of Instruction, Universities and Research of Italy. Technical support was provided by the Department of Energetics of Politecnico of Torino, Italy. Authors are also grateful to Giner Electrochemical Systems LLC. for providing the S-10 electrolyzer prototype to carry out this tests.

\section{References}

[1] Grigor'ev S. A., Khaliullin M. M., Kuleshov N. V., Fateev V. N., Electrolysis of Water in a System with a Solid Polymer Electrolyte at Elevated Pressure, Russian Journal of Electrochemistry, Vol. 37 (8), pp. 819-822, 2001.

[2] Shapiro D., Duffy J., Kimble M., Pien M., Solarpowered regenerative PEM electrolyzer/fuel cell system, Solar Energy, Vol. 79, pp. 544-550, 2005.

[3] Grigoriev S.A., Porembsky V.I., Fateev V.N., Pure hydrogen production by PEM electrolysis for hydrogen energy, International Journal of Hydrogen Energy, Vol. 31, pp. $171-175,2006$.

[4] Schug C.A., Operational characteristics of highpressure, high-efficiency water-hidrogen-electrolysis, International Journal of Hydrogen Energy, Vol. 23 (12), pp. 1113-1120, 1998

[5] Görgün H., Dinamik modelling of a proton exchange membrane (PEM) electrolyzer , International Journal of Hydrogen Energy, Vol. 31, pp. 29 - 38, 2006.

[6] Choi P., Bessarabov D.G., Datta R., A simple model for solid polymer electrolyte (SPE) water electrolysis, Solid State Ionics, Vol. 175, pp. 535-539, 2004.

[7] Onda K., Kyakuno T., Hattori K., Ito K., Prediction of production power for high-pressure hydrogen by highpressure water electrolysis, Journal of Power Sources, Vol. 132, pp. 64-70, 2004.

[8] Barbir F., PEM electrolysis for production of hydrogen from renewable energy sources, Solar Energy, Vol. 78, pp. 661-669, 2005.

[9] Marshall A., Børresen B., Hagen V., Tsypkin M., Tunold R., Hydrogen production by advanced proton exchange membrane (PEM) water electrolysersReduced energy consumption by improved electrocatalysis, Energy, Vol. 32, pp. 431-436, 2007.

[10] Roy A,, Watson S., Infield D., Comparison of electrical energy efficiency of atmospheric and high-pressure electrolysers, International Journal of Hydrogen Energy, Vol. 31, pp. 1964 - 1979, 2006. 
Nomenclature

$\mathrm{G}_{\text {H2O }} \quad$ Water flow $(1 / \mathrm{h})$

I Stack current (A)

I Current density $\left(\mathrm{A} / \mathrm{cm}^{2}\right)$

$\mathrm{P} \quad$ Cathode pressare (bar)

PEM Proton Exchange Membrane

$\mathrm{T} \quad$ Water inlet temperature $\left({ }^{\circ} \mathrm{C}\right)$

V Stack voltage (V) 